\title{
Arrested cerebellar development: a type of cerebellar degeneration in amaurotic idiocy ${ }^{1}$
}

\author{
REINHARD L. FRIEDE \\ From the Mental Health Research Institute and Department of Pathology, University of \\ Michigan, Ann Arbor, Michigan, U.S.A.
}

This paper reports on four cases of a specific type of cerebellar degeneration which suggests an arrest of cerebellar development at an early foetal stage. Histochemical and silver stain studies were necessary to demonstrate some of the more characteristic features which strikingly distinguished this type of degeneration from the granular layer type of cerebellar degeneration or the common types of cerebellar sclerosis.

All four cases were of amaurotic idiocy; but the type of cerebellar degeneration seemed to be characteristic by its onset at a certain developmental phase rather than characteristic of amaurotic idiocy in general. Possibly the same type of cerebellar degeneration involving developmental arrest also occurs in other diseases.

\section{MATERIAL AND METHODS}

The observations were made in four typical cases of infantile (Tay-Sachs type) amaurotic idiocy. The clinical and neuropathological findings were characteristic for the disease; the children died at the age of $3,3,6$, and 6 years. The present report will be restricted to the histological findings in the cerebellum, which was atrophic and of increased consistency in all cases.

The following staining methods were used: Haematoxylin-eosin, Klüver-Barerra, Bodian's silver impregnation, several modifications of Hortega's silver carbonate method, the periodic acid-Schiff reaction counterstained with chromalum gallocyanin (both in $30 \mu$ frozen and $10 \mu$ paraffin sections), Holzer's stain for glial fibrils, and the ninhydrin-Schiff reaction for proteins. Enzyme histochemical studies for diphosphopyridine nucleotide (D.P.N.) diaphorase, lactic dehydrogenase and alkaline, phosphatase were done for two cases; standardized methods for these enzymes have been described in detail elsewhere (Friede, Fleming, and Knoller, 1963).

\section{OBSERVATIONS}

The type of cerebellar change was identical in all four cases. The molecular layer was divided into an

${ }^{1}$ This investigation was partly supported by U.S. Public Health grant B-3250. outer and inner lamina. With haematoxylin-eosin and periodic acid-Schiff stains, these laminae were distinguished by almost complete absence of glial nuclei in the outer lamina and the homogeneous distribution of numerous glial cells in the inner lamina. Also, the outer lamina contained only a few capillaries which usually took a straight course, perpendicular to the surface, whereas in the inner lamina, capillaries were more numerous and contorted, often running parallel to the cortical surface. The difference in the capillary distribution was readily demonstrated in periodic acid-Schiff stains, which stain the basement membrane of the capillaries, and also by the reaction for alkaline phosphatase, which was very strong in capillaries. Another interesting observation was that lipid storage was restricted to the inner lamina (periodic acid-Schiff stains in frozen sections).

It should be noted that there was no difference in staining intensity of the ground substance of the laminae with either haematoxylin-eosin or periodic acid-Schiff stains, and that the differences in cell and capillary density were not easily noticed until studies with other stains alerted one to the fact that the molecular layer was actually divided into two laminae. The laminae were clearly distinguishable and obvious in studies with ninhydrin-Schiff, Klüver-Barerra, Bodian, and oxidative enzyme methods, all of which showed a definite difference in the staining intensity of the ground substance of the laminae.

With the Klüver-Barerra, Hortega, and Bodian stains the deep boundary of the outer lamina appeared as a sharp line because of numerous horizontally running cell processes in the outer lamina, often concentrated in a narrow band (Figs. 1, $2 b)$. This was in contrast to the inner lamina in which most of the cell processes took an irregular course. Holzer preparations demonstrated that there was no glial fibrosis in either layer; silver impregnations for astrocytes did not indicate astrocytic hypertrophy or proliferation in any part of the cortex or white matter. 


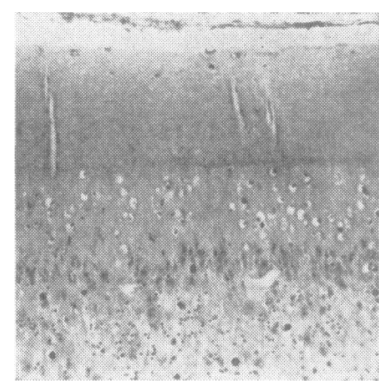

FIG. 1. Subdivision of the cerebellar molecular layer into an outer and inner lamina. Note the sharp separation of the laminae by a horizontal band of glial fibres and the marked difference of the number of glial cells in these laminae.

Klüver-Barrera, $\times 100$.

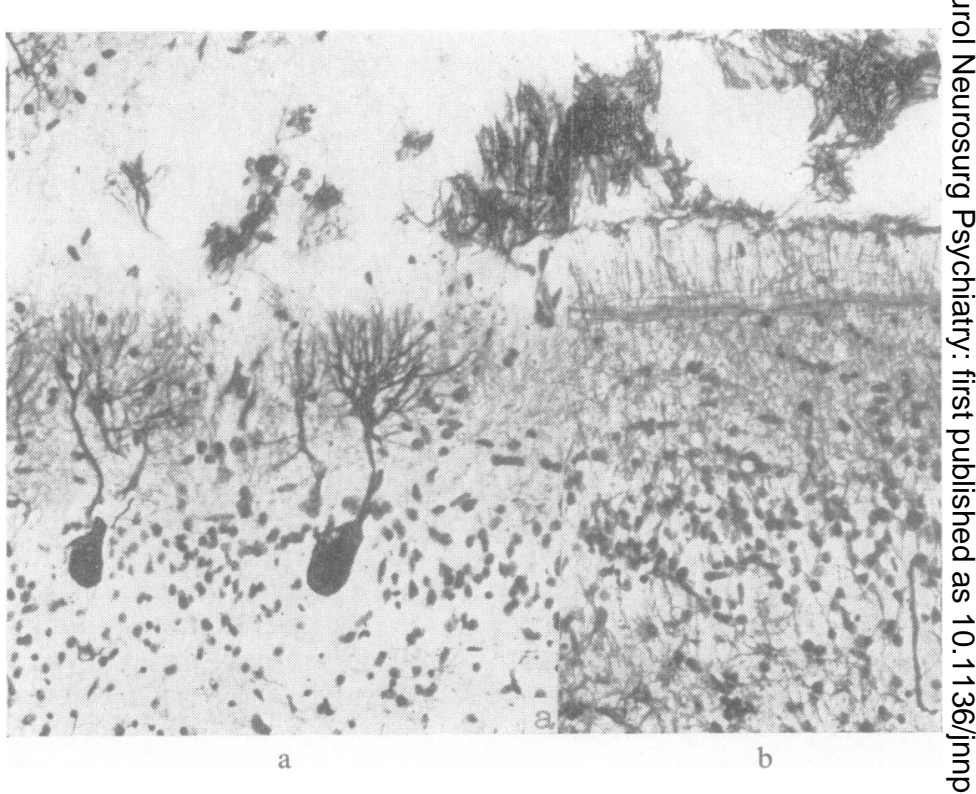

FIGS. 2a and 2b. Sections stained with modifications of Hortega's silver $\mathrm{N}$ carbonate method to show (a) the sharp termination of Purkinje dendrites - at the outer lamina and (b) the architecture of glial fibres in the same regiog. I The outer lamina in this case was relatively thin. $\times 150$.

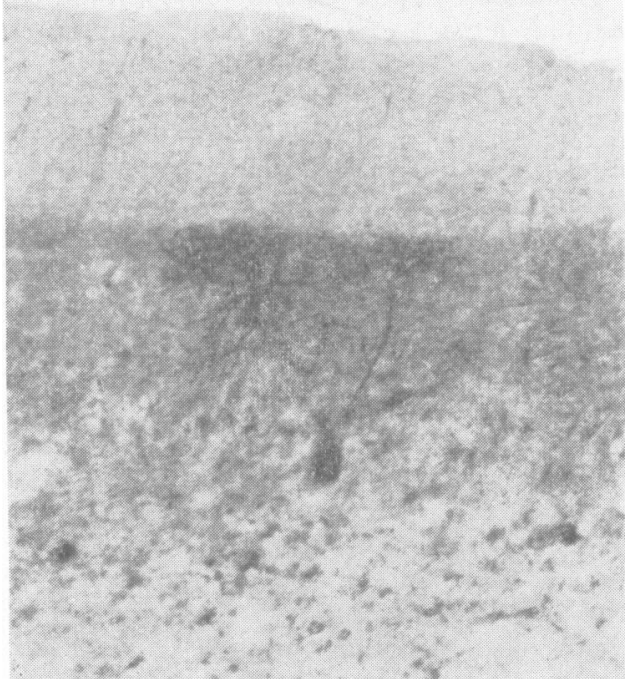

FIG. 3

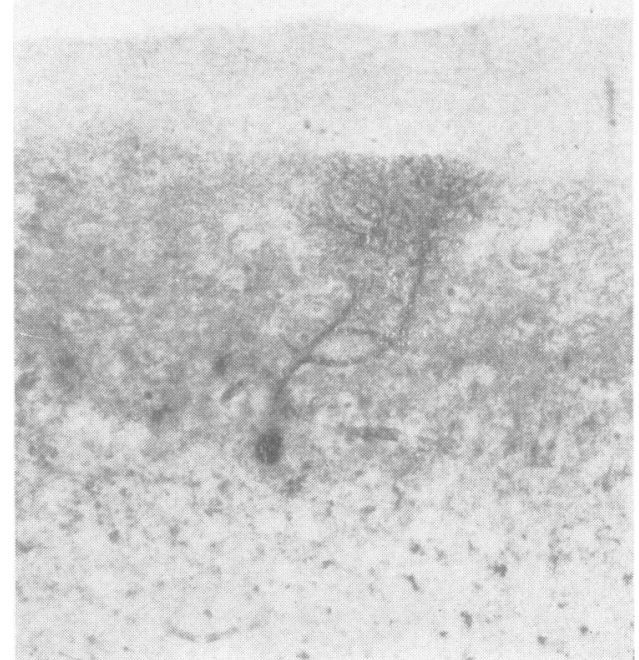

FIG. 4

FIGS. 3 and 4. Histochemical reaction for D.P.N.-diaphorase. There is almost no enzyme reaction in the outer lamina and a diffuse, weak reaction in the inner lamina. The dendrites of the Purkinje cells are demonstrated by a strong reaction; they terminate, as if cut off, at the border of the laminae. $\times 170$. 


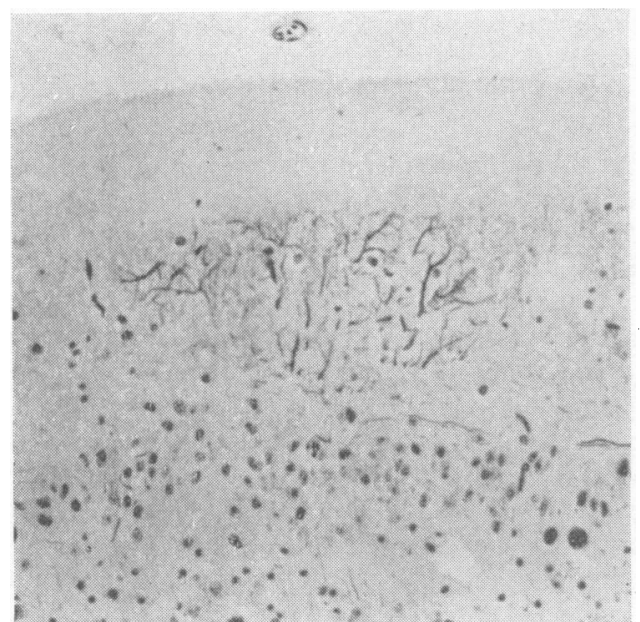

FIG. 5

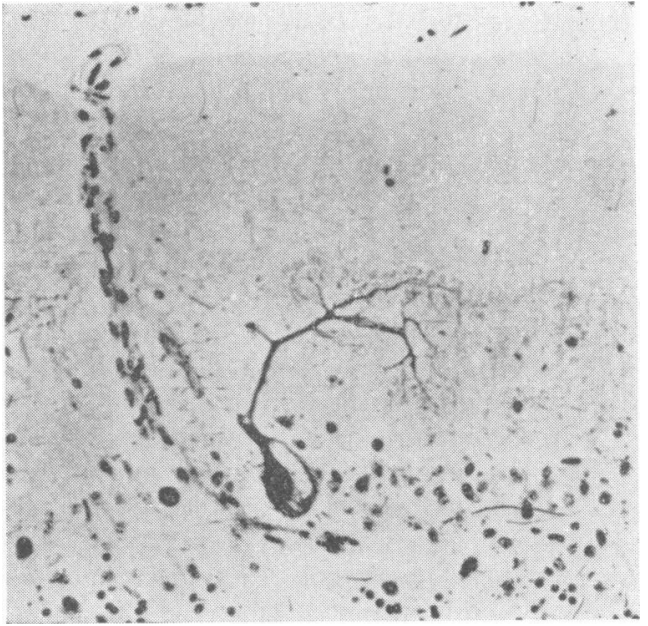

FIG. 6

FIGs. 5 and 6. Paraffin sections stained with Bodian's silver stain demonstrate the termination of dendrites at the border of the laminae. $\times 170$.

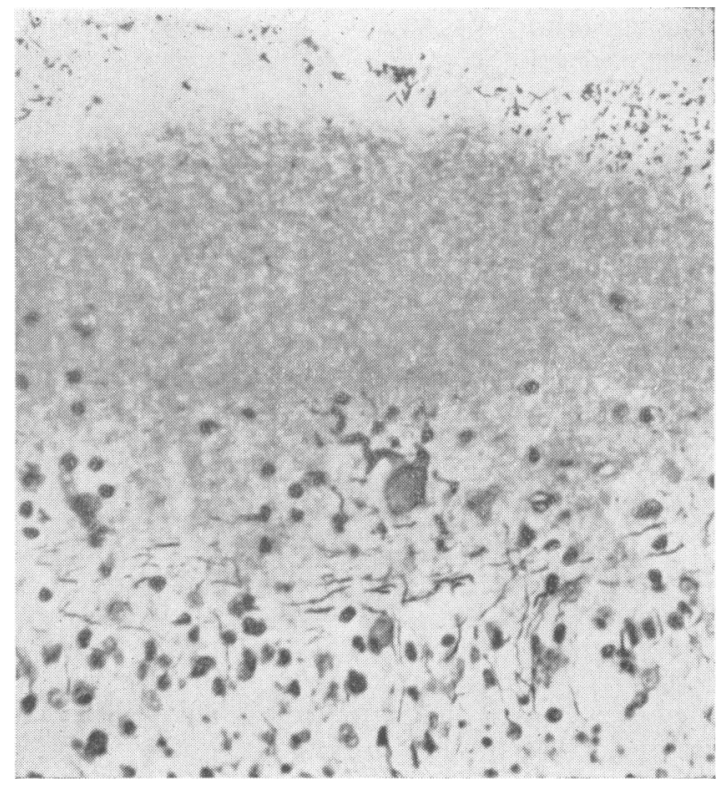

FIG. 7

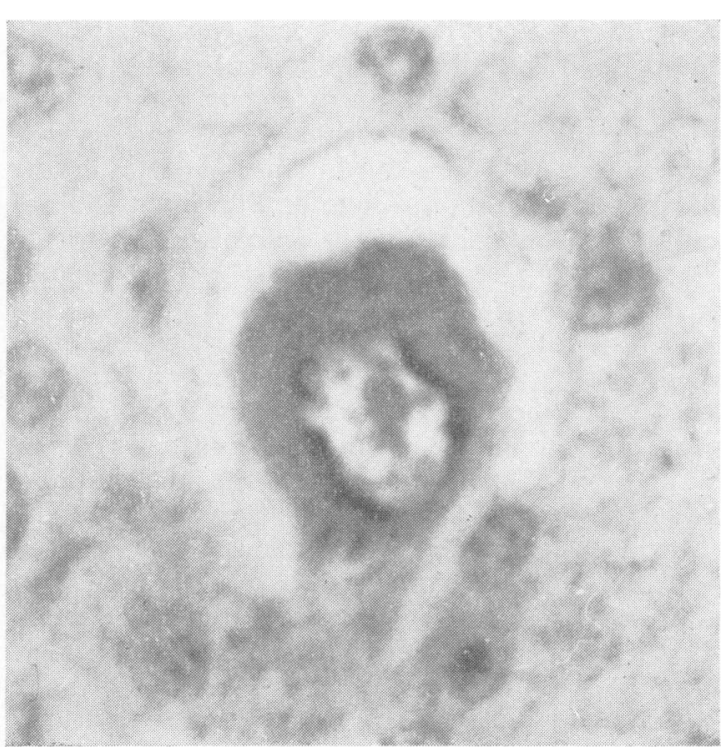

FIG. 8

FIG. 7. Very thick outer lamina; the plump and contorted dendrites of a Purkinje cell are confined to the thin inner lamina. Note the gradient of cell density as compared with Figure 1. Bodian's silver stain. $\times 250$.

FIG. 8. Thickening and hyperchromasia of the nuclear membrane of a Purkinje cell. Chromalum-gallocyanin. $\times 800$. 
The most characteristic feature of the disease was the arrangement of Purkinje dendrites, which were present only in the inner lamina. Wherever the dendritic arborizations of Purkinje cells approached the borderline between inner and outer lamina, they terminated sharply at the outer lamina, as if cut off along a straight line (Figs. 2a, 3-6). This was independent of whether the dendrites originated from the cell body or, secondarily, from one of the so-called asteroid bodies in the molecular layer. Rarely, an occasional dendrite branch was observed in the outer lamina for a short distance.

The sharp separation into laminae was most impressively demonstrated in histochemical preparations for D.P.N.-diaphorase and lactic dehydrogenase. There was practically no enzyme reaction in the outer lamina, while a weak reaction was homogeneously distributed in the inner lamina. This pattern of enzyme distribution was consistent, regardless of the presence or absence of Purkinje cells at a given site. Very little enzyme activity was seen in other parts of the cortex. All the dendritic arborizations of the Purkinje cells were stained by a marked reaction for D.P.N.-diaphorase and lactic dehydrogenase, and could be seen to terminate abruptly at the boundary between the laminae (Figs. 3 and 4). Also, there was an extremely strong enzyme reaction in the so-called asteroid bodies.

The thickness of the outer lamina was different for each case. While there were regional variations, the outer lamina occupied approximately two-thirds of the molecular layer in one case (Fig. 7) and approximately one-half, one-third, and one-fifth in the other cases. The branchings of Purkinje dendrites and the presence of glial cells were always restricted to the inner lamina, regardless of its relative thickness.

Cytologically, Purkinje cell nucleoli were weakly stained with chromalum gallocyanin in contrast to the nuclear membranes which stained darker than usual and showed attached caps of chromatin (Fig. 8). In normal control material, the nucleoli were stained darker than the cell membranes.

In all four cases, there was considerable loss of both Purkinje and granule cells, and the persisting granule cells were often grouped in a patchy pattern. Myelination was absent in three of the cases and incomplete in the fourth.

\section{DISCUSSION}

The thickness and the gradual involution of the foetal external granular layer has been studied by Berliner (1905). Before the fifth foetal month, the external granular layer occupies more than half of the primitive molecular layer; between the sixth and the ninth months, it is gradually reduced to roughly one-fifth of the molecular layer. It persists at birth, being approximately five to eight cells thick. After the third post-natal month, the speed of reduction is accelerated and the external granular layer normally disappears by the ninth post-natal month (Biach, 1910).

In our cases, the architecture of the cerebellar cortex was like that seen in foetal cerebellar cortex at the stage of development when a thick external granular layer is present. The dendrites of Purkinje cells extended only as far as they would if this layer were still present, that is, to the border of the external granular layer (Berliner, 1905). Thus the cortical architecture looked as if it had been halted or 'frozen' at an early developmental phase. The cells of the foetal external granular layer had disappeared, but the space previously occupied by this layer persisted as a distinct 'outer lamina'; it was almost without cells, poor in vascularization, and devoid of oxidative enzyme activity.

In the three cases with the thicker outer lamina, there was no myelination in the white matter and the internal granular layer was poorly developed. This suggested that both lack of myelination and the so-called 'degeneration' of the internal granulars layer might not have been a true atrophy, that is,, $\mathbb{Q}$ reduction of a fully developed layer, but rather ang arrest of development. At the phase of developmento when the external granular layer is very thick, thereo is no myelination in the white matter and the internal granular layer is poorly developed. In the fourth case, the outer lamina occupied about one-fifth of the molecular layer, thus suggesting a phase of development close to birth. In this case, the white matter was partly myelinated and the degree of myelination seemed to agree with what one would expect at a stage when the external granular layer would have been the thickness of the outer lamina in this case. Myelination normally proceeds from the cerebellar nuclei toward the periphery and is not completed until after birth (de Sanctis, 1898).

Further support for the concept of arrested development may be derived from comparison with the development of oxidative enzyme patterns in rat cerebellar cortex (Friede, 1957). Between the seventh and tenth day of rat post-natal development, which is homologous to a human foetal state, oxidative enzyme activity in the cerebellum is restricted to the perikarya of Purkinje cells and their primitive dendritic arborization which are present only in the inner portion of the molecular layer; there is little activity in the rest of the cortex. The enzyme pattern in our cases so closely resembled the enzyme pattern of foetal cerebellar cortex that the pathology 
appeared to have involved lack of development rather than loss of enzyme activity.

All the features described above suggest that the development of the cerebellar cortex was arrested at an early phase. The only conceivable alternative explanation we could think of was that an intense gliosis in the outer portion of the molecular layer might have acted as a barrier for the growth of Purkinje dendrites. This was ruled out by Holzer stains which gave no indication of a selective fibrillary gliosis of the outer layer. The almost complete absence of glial nuclei from the outer layer also contradicts this concept.

Developmental arrest evidently is not diagnostic for amaurotic idiocy but it may well be diagnostic for the time of the onset of the metabolic disorder. Accordingly, we did not find arrested cerebellar development in one juvenile case of amaurotic idiocy. Illustrations by Bielschowsky $(1913,1920)$ suggest that this type of pathology has been seen before; a paper by Westphal (1917) mentions the gradient of cell density of the two laminae of the molecular layer, but both authors considered the outer lamina as an area of gliosis. There seems to be some overlap of arrested development with the granular layer type of cerebellar atrophy (Schob, 1921; Scherer, 1933; Norman, 1940; Jervis, 1950; Ule, 1952); however, arrested cerebellar development should be considered an entity because of its implications on the timing rather than the nature of the disorder. Exactly how accurate such a timing can be is difficult to assess because delayed involution of the external granular layer has been observed in a variety of conditions (Biach, 1910; Lotmar, 1931).

\section{SUMMARY}

A description is presented of a specific type of cerebellar degeneration observed in four cases of infantile amaurotic idiocy. The cerebellar architecture strongly suggested that there had been an arrest of cerebellar development at an early foetal stage. The molecular layer was divided into two laminae; the outer of these replaced the foetal external granular layer but contained almost no cells and very few capillaries. The Purkinje cell dendritic arborizations were restricted to the inner lamina and terminated, as if cut off, at the border between the two laminae. There was a lack of development of the internal granular layer and deficient myelination.

\section{REFERENCES}

Berliner, K. (1905). Arch. mikr. Anat., 66, 220.

Biach, P. (1910). Arb. neurol. Inst. Univ. Wien, 18, 13. Bielschowsky, M. (1913). Disch. Z. Nervenheilk., 50, 7. - (1920), J. Psychol. Neurol. (Lpz.), 26, 123

de Sanctis, S. (1898). Mschr. Psychiat. Neurol., 4, 237.

Friede, R. (1957). Arch. Psychiat. Nervenkr. 196, 196.

_- Fleming, L. M., and Knoller, M. (1963). J. Histochem. Cytochem. 11, 232.

Jervis, G. A. (1950). J. nerv. ment. Dis., 111, 398.

Lotmar, F. (1931). Z. ges. Neurol. Psychiat., 136, 412.

Norman, R. M. (1940). Brain, 63, 365.

Scherer, H. J. (1933). Z. ges. Neurol. Psychiat., 145, 335.

Schob, F. (1921). Ibid., 73, 188.

Ule, G. (1952). Dtsch. Z. Nervenheilk., 168, 195.

Westphal, A. (1917). Arch. Psychiat. Nervenkr., 58, 248. 